\title{
Innovative Approaches to Assess the Impact and Steps to Decrease the Neurological Consequences of Ablation
}

\author{
SHIVA P. PONAMGI, MD ${ }^{1}$, OMAR Z. YASIN, MD, MS ${ }^{2}$, DEEPAK PADMANABHAN, $\mathrm{MD}^{3}$, \\ CHRISTOPHER V. DESIMONE, MD, PhD ${ }^{3}$ and SAMUEL J. ASIRVATHAM, MD ${ }^{3,4}$ \\ ${ }^{1}$ Division of Hospital Internal Medicine, Mayo Clinic Health System, Austin, MN \\ ${ }^{2}$ Division of Internal Medicine, Mayo Clinic, Rochester, MN \\ ${ }^{3}$ Division of Cardiovascular Diseases, Mayo Clinic, Rochester, MN \\ ${ }^{4}$ Department of Pediatrics and Adolescent Medicine, Mayo Clinic, Rochester, MN
}

\begin{abstract}
Despite continued innovations in catheter design and technique, catheter ablation for atrial fibrillation $(A F)$ is still fraught with a few serious complications-most feared of which is stroke. Although a "classic" clinically disabling stroke is less common (about 1\%) after AF ablation, many recent studies have identified an increased incidence of asymptomatic ischemic cerebral lesions known as "silent clinical lesions" (SCLs) associated with the procedure. As once thought, these new SCLs seen on post-ablation magnetic resonance imaging of the brain were not actually clinically "silent" and were shown to have significant clinical and neuropsychiatric effects on these patients in the long term. These SCLs are thought to represent the "embolic fingerprints" of the ablation procedure, suggesting involvement and the need for innovation as well as improved safety at multiple levels before, during, and after the procedure. This may require a multimodality approach involving several measures such as having better peri-procedural anticoagulation strategies, using real-time monitoring for markers of neurologic injury, giving meticulous attention to sheath management, using novel energy sources that are less thrombogenic, and finally establishing imaging protocols for timely detection of these lesions post ablation. The current literature is reviewed here to explore such opportunities to improve neurological outcomes of catheter ablation for AF.
\end{abstract}

KEYWORDS. Ablation, atrial fibrillation, silent cerebral lesion/event, stroke.
ISSN 2156-3977 (print) ISSN 2156-3993 (online)

(C) 2016 Innovations in Cardiac Rhythm Management

\section{Introduction}

Atrial fibrillation (AF) is a major health-care problem with profound economic and public health implications. ${ }^{1}$ A systematic review of population-based studies of AF worldwide estimates a prevalence of 33.5 million cases of

S. J. Asirvatham is a consultant with Abiomed, Atricure, Biosense Webster, Biotronik, Boston Scientific, Medtronic, Spectranetics, St. Jude, Sanofi-Aventis, Wolters Kluwer, Elsevier, and Zoll.

Manuscript received March 16, 2016, Final version accepted April $12,2016$.

Address correspondence to: Samuel J. Asirvatham, MD, Professor of Medicine and Pediatrics, Division of Cardiovascular Diseases and Department of Pediatrics and Adolescent Medicine Mayo Clinic, 200 First Street SW, Rochester, MN 55905, USA.

E-mail: Asirvatham.samuel@mayo.edu
AF as of 2010, with an additional 5 million new cases being reported every year. ${ }^{2}$ Radiofrequency catheter ablation (RFA) for AF is an effective therapeutic option for symptomatic drug refractory $\mathrm{AF}^{3-5}$ However, this procedure is still riddled with the most feared complication of AF ablation: neurologic injury.

Neurologic injury during AF ablation can be devastating and irreversible. In this paper, we have reviewed current literature exploring the technologies being used to detect neurologic injury and the risk factors associated with this injury. We have also identified potential areas of intervention that could lower this risk such as 1) adopting stringent procedural safety techniques, 2) using uninterrupted warfarin or interrupted anticoagulation with novel oral anticoagulants (NOACs) for 
peri-procedural anticoagulation, 3) using novel energy sources and catheters, and 4) using real-time markers for potential neurologic injury. We also highlight areas of uncertainty requiring more research or innovation.

\section{Silent cerebral lesions or events}

Although disabling stroke associated with AF ablation is rare (about $1 \%$ of cases), ${ }^{6}$ recently, silent cerebral lesions or events (SCLs or SCEs) are increasingly recognized as a more common and extremely worrisome complication of AF ablation. The incidence of SCLs/SCEs in clinical studies is estimated to be about 100 times more than symptomatic cerebral ischemia. ${ }^{7}$ While new ischemic brain lesions on magnetic resonance imaging (MRI) have been reported in up to $50 \%$ of post-AF ablation patients, ${ }^{8-11}$ SCLs/SCEs (independent of the MRI-specific definition used) have been reported only in $12.6 \%$ of them. ${ }^{7}$

Haines et al. ${ }^{12}$ suggested that the microbubbles and microembolic debris seen in in vivo swine models may in fact represent thermal coagulum formed due to excessive heating and increased current density caused by the overlapping of the 1 and 10 electrodes of the pulmonary vein ablation catheter (PVAC). This debris could embolize during or immediately after the procedure, showing up as new lesions on the MRI done post-AF ablation. In fact, direct carotid injections of high volumes of air mixes or thrombotic debris mixes in canine models showed similar MRI findings, suggestive of SCLs/SCEs that were independent of the type of materials used., ${ }^{72}$

SCL/SCE is defined as an acute new MRI-detected brain lesion typical of cerebral ischemia in a patient without any clinically apparent neurological deficits. ${ }^{7}$ However, it is important to recognize the heterogeneity and the lack of clear consensus among different investigators in regard to defining these SCLs/SCEs. While some groups have defined the "new MRI-detected lesion" as a hyperintense diffusion-weighted imaging (DWI) lesion with reduced apparent diffusion coefficient (ADC) and T2-weighted fluid-attenuated inverse recovery sequence
(FLAIR) positivity, ${ }^{10,13-17}$ a few others have used a more sensitive definition without the FLAIR positivity. ${ }^{11,18-26}$ There is much controversy regarding the significance of SCLs/SCEs and their long-term consequences. In addition to the structural MRI findings, several recent studies have suggested a strong association with function, correlating SCLs/SCEs and cognitive decline, as well as dementia. $^{27-29}$ There is some credence to the structural/ functional correlation added by a study led by Medi et al., ${ }^{30}$ which showed the prevalence of post-AF ablation cognitive dysfunction being $27-28 \%$ at $24-48$ hours and $13-20 \%$ at 90 days after the procedure. Interestingly, in this study the length of time of ablation within the left atrium was associated with cognitive dysfunction. ${ }^{30}$ This could be reflective of more chance for coagulum to occur in the left-sided circulation and thus lead to SCLs/SCEs and/or thromboembolism.

\section{Imaging for neurological consequences of ablation: detecting SCLS/SCEs}

MRI of the brain: sequences, resolution, and timing SCLs/SCEs may have significant consequences including cognitive decline and dementia. ${ }^{28,29}$ MRI imaging using a FLAIR technique can take up to $20 \mathrm{~h}$ to detect neurologic infarction and can take even longer for smaller infarcts. ${ }^{31}$ This makes it challenging for FLAIR to be useful in SCL/ SCE detection immediately after AF ablation. In contrast, MRI using DWI can detect even small infarcts within minutes of their development, as this sequence is precise on picking up cellular edema (one of the early manifestations of ischemic cell injury) and shows up as hyperintense lesions on MRI (Figure 1). ${ }^{32,33}$ A study by Wieczorek et al. ${ }^{25}$ highlight the difference in timing and sequences; they have shown that only $33 \%$ of patients with DWI positive lesions had FLAIR positivity on MRI imaging done the day following ablation. ${ }^{23}$ Therefore, the use of DWI sequences is currently considered the cornerstone for diagnosing cerebral ischemia especially
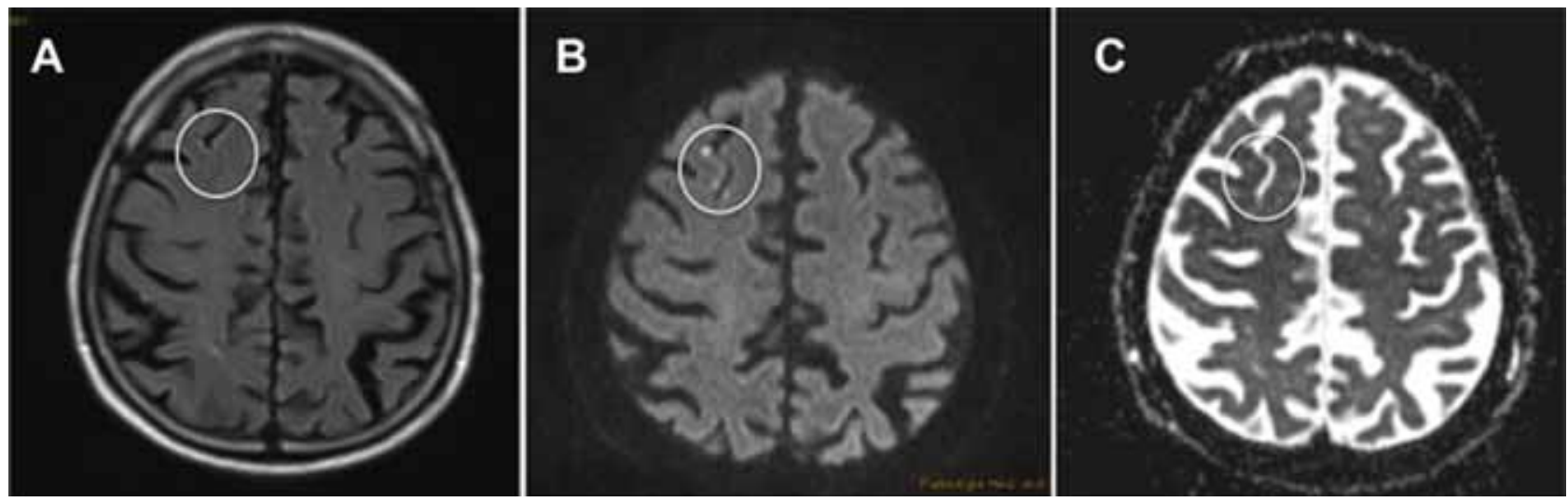

Figure 1: Magnetic resonance imaging 1 day after atrial fibrillation ablation using (a) T2-weighted fluid-attenuated inverse recovery (FLAIR) sequence, (b) diffusion-weighted imaging (DWI), and (c) apparent diffusion coefficient (ADC) mapping. Three acute lesions are identified on DWI (circle) with corresponding reduced ADC (circle) but no FLAIR positivity (circle). These lesions are called silent cerebral events (SCE) and detection is more sensitive to acute cerebral ischemia than adding FLAIR positivity. Reproduced with permission from the Journal of Cardiovascular Electrophysiology. ${ }^{7}$ 
small SCLs/SCEs that may develop immediately after AF ablation procedure.

The size of SCLs/SCEs seen on MRI post ablation seem to vary widely in different studies, ranging from $2.5 \mathrm{~mm}$ to $35 \mathrm{~mm}$, with more than $50 \%$ of them measuring less than $3 \mathrm{~mm} .{ }^{7,20,34,35}$ A small proportion of the lesions seem to be larger than $10 \mathrm{~mm}$ and seen predominantly in the cerebellum. $^{8-11,13,14,18,21,36-38}$ Although most studies reporting post-AF ablation SCLs/SCEs were performed using 1.5-Tesla MRIs, one study using a 3-Tesla high-resolution MRI reported a two to three times increase in the incidence of the lesions in patients undergoing left atrial catheter ablation independent of the respective balloon-based catheter devices. The new lesions seen in this study were not associated with cognitive decline in patients after the ablation, suggesting a mere increase in the imaging sensitivity rendered by the higher spatial resolution rather than increased cerebral embolization during the procedure. $^{10}$

The best time window to evaluate for post ablation SCLs/SCEs using MRI continues to be a hotly debated topic. Although DWI can detect lesions within $15 \mathrm{~min}$ of the procedure, they can also disappear in 2-4 days. ${ }^{7}$ Alternatively, FLAIR sequences may not be able to consistently detect these lesions until day 2. Further complicating the interpretation is that some studies have shown that these lesions may be attenuated over time, with no discernable lesions noticed on repeat MRIs done at 21 months $^{21}$ and 1 year ${ }^{11}$ of follow-up. These findings were also confirmed in a later study using 3-Tesla MRI. ${ }^{10}$ Therefore, it is considered to be most practical to evaluate within $24 \mathrm{~h}$ if using a DWI sequencing technique, but it may also be prudent to wait for about 2-7 days if using FLAIR sequencing. ${ }^{7}$

In most of the studies to date, post-ablation MRI to evaluate for stroke or SCLs/SCEs was performed within 48-72 h of the ablation procedure. Although no particular location was shown to be significantly more prone, some studies suggested that there might be a predilection for the middle cerebral artery ${ }^{18,10}$ or the vertebral artery territories. ${ }^{8,9,11,13,21}$ Importantly, this is consistent with the most common patterns seen in embolic strokes. Thus, SCLs/SCEs that have been detected on MRI post ablation have been suggested to be the "embolic fingerprint" of all the steps or "missteps" that occur during a procedure due to their unique pattern and propensity for neurologic consequence. Hence these "embolic fingerprints" of a specific cardiovascular intervention $8,14,35,39$ could potentially be used evaluate the impact of novel ablation techniques or even modify the steps in existing procedures to improve safety and decrease their risk. ${ }^{7}$

\section{Approaches to decrease the impact for neurological consequences of ablation}

Pre-procedural measures

Underlying arrhythmia: Sinus rhythm versus atrial fibrillation Not surprisingly, long-standing persistent atrial fibrillation (AF) has been shown to have greater chances of having SCLs/SCEs during the course of the ablation procedure than paroxysmal atrial fibrillation or supraventricular tachycardia (SVT) ${ }^{40}$ This is logical, as the time of the procedures and amount of ablation are indeed going to be more extensive in patients with persistent AF. Furthermore, ablation for atrial fibrillation has greater chances for incidence of SCLs/SCEs than ablation for supraventricular arrhythmias. ${ }^{30}$ This might reflect the requirement of accessing the left-sided circulation in all AF ablation cases.

Imaging

Transesophageal echocardiography. Pre-procedural transesophageal echocardiography (TEE) is routinely performed to screen for any intracardiac thrombus, and its presence is an absolute contraindication for any cardiac ablation procedure. ${ }^{41}$ TEE is considered both a sensitive and specific imaging modality for detecting several cardiogenic or aortogenic thromboembolic sources. ${ }^{42}$ This imaging is critical to lower the risk of events prior to reaching the EP laboratory. More recently, in studies using TEE, a close correlation has been found between left atrial abnormalities and complex aortic arch plaques detected in neurologically asymptomatic patients who undergo AF ablation. The presence of new SCLs/SCEs on brain MRI may be suggestive of microembolization. ${ }^{43}$ Intuitively, there is a possibility of increased thromboembolism in patients undergoing ablation using a retro-aortic route, especially in the presence of atherosclerotic plaque in the aorta. Spontaneous echo contrast (SEC) as visualized by TEE has also been known to increase risks of intraprocedural thromboembolism in AF ablation. ${ }^{44}$ Thus, this pre-procedural screening imaging to evaluate for sources of intracardiac thrombi or aortic plaques is necessary to minimize the risk for thromboembolism.

Pre-procedural anticoagulation

Uninterrupted versus interrupted warfarin strategy. There is much controversy regarding the proper strategy for peri-procedural anticoagulation for AF ablation. There is evidence to suggest that uninterrupted warfarin therapy lessens the incidence of serious bleeds or embolic phenomena compared to bridging with heparin. ${ }^{45-47}$ Data from a prospective study of more than 6,000 patients by Di Biase et al. ${ }^{48}$ suggested a decreased incidence of thromboembolic events in patients undergoing AF ablation with the use of an irrigated RF catheter under uninterrupted warfarin anticoagulation with a therapeutic international normalized ratio (INR) on the day of the procedure.

The COMPARE trial was an open-label, randomized controlled trial of 1,584 patients, which found no significant difference in the incidence of major bleeding and pericardial effusions between patients who were continued on or were taken off warfarin prior to ablation. ${ }^{45} \mathrm{~A}$ meta-analysis by Santangeli et al. $^{49}$ also supported the benefit of periprocedural anticoagulation with warfarin, as they found this had decreased the risk of thromboembolic complications when warfarin was continued and no statistically significant risk of increased bleeding.

These findings have also been examined with the use of MRI and correlated with SCLs/SCEs. The incidence of SCLs/ SCEs was found to be lower in patients undergoing RFA with uninterrupted warfarin therapy. In an observational study by Di Biase et al., ${ }^{50}$ pre- and post-procedural MRI was used to estimate the incidence of SCLs/SCEs, and the authors showed that sub-therapeutic INR before the procedure was associated with an increased risk of SCLs/ SCEs compared with uninterrupted warfarin therapy with a bolus of heparin prior to transseptal puncture. The 
increased risk of SCLs/SCEs was also associated with two consecutive measurements of activated clotting time (ACT) levels $<300$, as well as the failure to receive a loading dose of heparin. ${ }^{50}$

Anticoagulation with novel oral anticoagulants. Several studies have compared the use of novel anticoagulants during AF ablation. These studies assessed the prevention of thromboembolism and bleeding risks while using dabigatran, rivaroxaban, or apixaban. However, there are a limited number of randomized control trials comparing NOACs with uninterrupted warfarin therapy. Current studies do not suggest the benefit of any of the individual NOACs over uninterrupted warfarin therapy. ${ }^{51-54}$ Thus, the question remains whether uninterrupted NOACs are inferior to uninterrupted warfarin therapy for ablation. The risks of increased bleeding and the concern of controlling major bleeding in the event of ablation complications, remains a "sword of Damocles," especially in the absence of any effective and approved antidotes to the NOACs.

Dabigatran: Some studies suggested similar risks of symptomatic thromboembolism and bleeding with dabigatran use versus uninterrupted warfarin. Other studies have suggested an increased risk of bleeding and increased incidence of SCLs/SCEs. In a prospective observational study by Lakkireddy et al., ${ }^{51}$ patients who were on peri-procedural dabigatran for AF ablation (dose held on the morning of the procedure) were compared with matched patients undergoing AF ablation with uninterrupted warfarin therapy. There was no statistically significant difference in the incidence of the thromboembolic events found between the two groups. However, the data suggested a significant increase in the rates of major bleeding in the dabigatran group. ${ }^{51}$ Several other studies suggested that there was no significant difference in thromboembolic events or incidence of major bleeding between warfarin and dabigatran groups and this was further corroborated by subsequent metaanalyses. ${ }^{55-61}$ Two observational studies focused on the rates of SCLs/SCEs in patients treated with dabigatran versus uninterrupted warfarin and showed increased incidence of SCLs/SCEs detected on MRI in the dabigatran group compared with patients on warfarin with statistically significant increased risk of hemopericardium treated with pericardiocentesis in one of the studies. ${ }^{62,63}$

Rivaroxaban: Uninterrupted rivaroxaban use was found to have similar outcomes to those of uninterrupted warfarin in terms of symptomatic thromboembolism, bleeding risk, and incidence of SCLs/SCEs. Two separate studies found no statistically significant difference in the incidence of embolic events or major bleeding complications between uninterrupted rivaroxaban and uninterrupted warfarin in patients undergoing AF ablation. ${ }^{52,64}$ Two separate meta-analyses showed no statistically significant difference in the risk of thromboembolism or incidence of major bleeding in patients on uninterrupted rivaroxaban compared with uninterrupted vitamin $\mathrm{K}$ antagonists. ${ }^{65,66}$ Nakamura et al. ${ }^{63}$ assessed the risk of SCLs/SCEs when using rivaroxaban compared with other anticoagulants but did not find an increased risk of SCLs/SCEs on multivariate analysis when rivaroxaban was used $(\mathrm{p}=0.324)$.

Apixaban: Apixaban has similar rates of thromboembolism and SCLs/SCEs to uninterrupted warfarin use. In a prospective observational study by Di Biase et al., ${ }^{54}$ where the authors compared patients with uninterrupted apixaban use to those with uninterrupted warfarin therapy, no symptomatic thromboembolic complications or SCLs/SCEs on MRI occurred in either group. In addition, the rates of major and minor complications were comparable. Another randomized controlled trial where patients were randomized to uninterrupted warfarin versus uninterrupted apixaban with MRI performed in all patients before and after ablation showed similar rates of SCLs/SCEs and other complications in both groups. ${ }^{67}$ Moreover, in the multivariate analysis by Nakamura et al. ${ }^{63}$ there was no statistically significantly increased risk of SCLs/SCEs when using apixaban $(\mathrm{p}=0.090)$.

In summary, there is clear benefit from uninterrupted anticoagulation with warfarin in reducing the risk of thromboembolism. Novel anticoagulants have similar outcomes on symptomatic thromboembolism to that of warfarin. Apixaban and rivaroxaban in particular have evidence suggesting similar risk of SCLs/SCEs to that of warfarin. Current evidence suggests that an increased incidence of SCLs/SCEs is found when dabigatran is used. More randomized controlled trials are needed to assess the risk of SCLs/SCEs when using NOACs, and more uniform protocols for periprocedural NOAC management should be adopted to allow for cross comparison of studies.

\section{Intraprocedural measures}

Imaging

Real-time monitoring using transcranial Doppler (TCD) and intracardiac echocardiogram (ICE) may provide an inherent advantage of titrating RF energy during the procedure to reduce ablation and re-assess ACT. In contrast, MRI is limited as this is only performed after the procedure when the damage has already been done. These imaging modalities may be important in identifying small thrombi around the implanted leads or the prosthetic devices and develop innovative strategies to prevent thromboembolism in real time. This may in turn reduce the risk of SCLs/SCEs during AF ablation and improve the long-term outcomes associated with it.

Transcranial Doppler. TCD is a well-established technique that has been used in a variety of clinical settings to detect microembolic signals (MESs) in the cerebral circulation. ${ }^{6-74}$ It could be used to detect MESs of both gaseous and solid nature, and hence has been used successfully in many studies for monitoring cerebral MESs during different types of endocardial and epicardial ablation procedures (Figure 2). ${ }^{74-78}$ Monitoring the MES count with TCD may even be used to gauge SCL/SCE risk during an ablation procedure. ${ }^{79}$ This would have major ramifications on real-time reduction in embolic risk during procedures. However, it is important to note that several studies have used different identification criteria for embolic signals detected by TCD leading to some differences in reported frequencies of embolic signals. Importantly, this has led to the development of criteria by different consensus committees to bring uniformity to these measurements. ${ }^{80,81}$ Dittrich et al. ${ }^{82}$ showed that standardized MES readings by TCD can be used as a robust and a surrogate marker in clinical trials as long as independent quality control mechanisms are in place.

Intracardiac echocardiography. In addition to prophylactic imaging prior to the actual procedure, ICE also provides realtime feedback regarding lesion formation and potential information regarding microbubble formation. Excessive tissue heating to the point of steam formation during RF ablation can lead to microbubble formation (Figure 3). ${ }^{83}$ Kilicaslan et al. ${ }^{76}$ and Nagiy-Baló et al. ${ }^{79}$ showed that there was a close correlation between the number of MESs detected during the ablation procedure and the degree of microbubble formation seen on ICE (Figure 4). In addition, studies have suggested an increased intensity anticoagulation strategy 


\section{Embolic shower Type I}

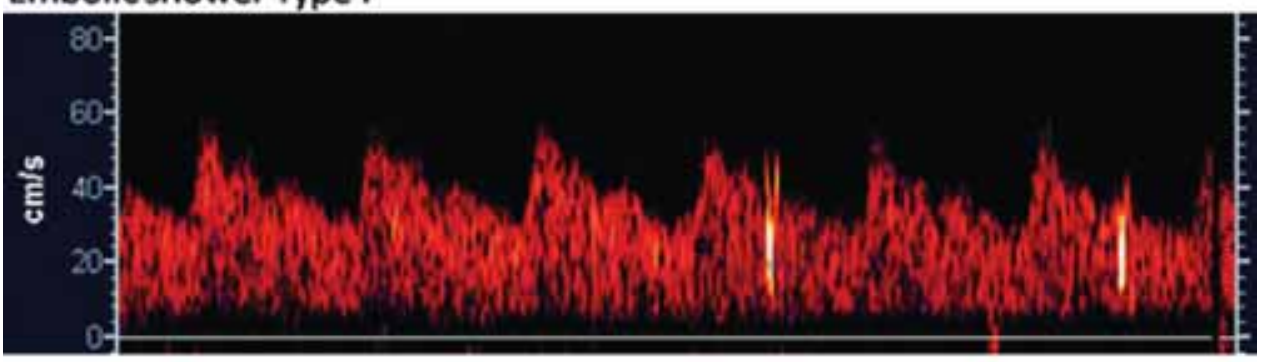

Embolic shower Type II

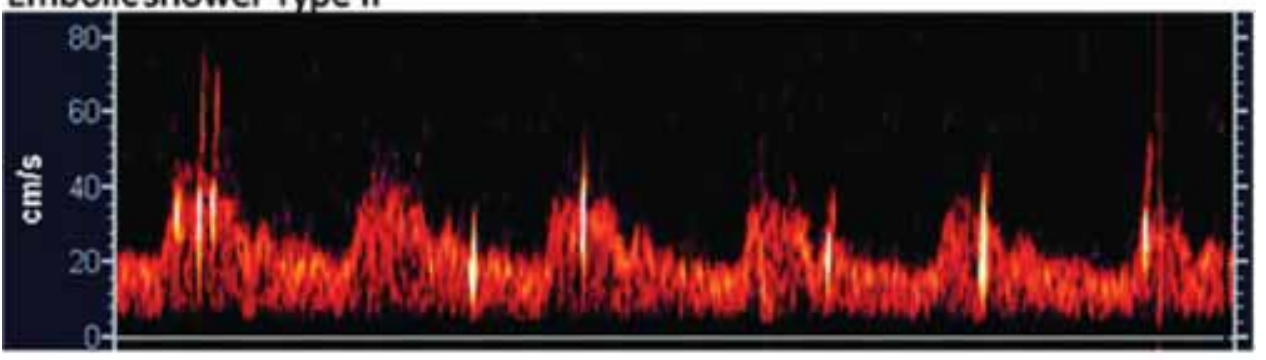

Embolic shower Type III

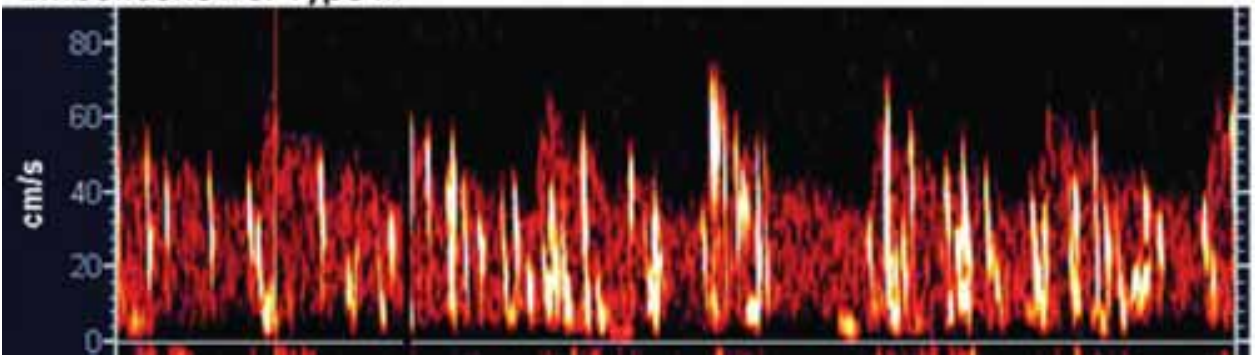

Figure 2: Transcranial measurement of cerebral microembolic signals during endocardial pulmonary vein isolation. Comparison of three different ablation techniques. Reproduced with permission from the Journal of Cardiovascular Electrophysiology. ${ }^{74}$

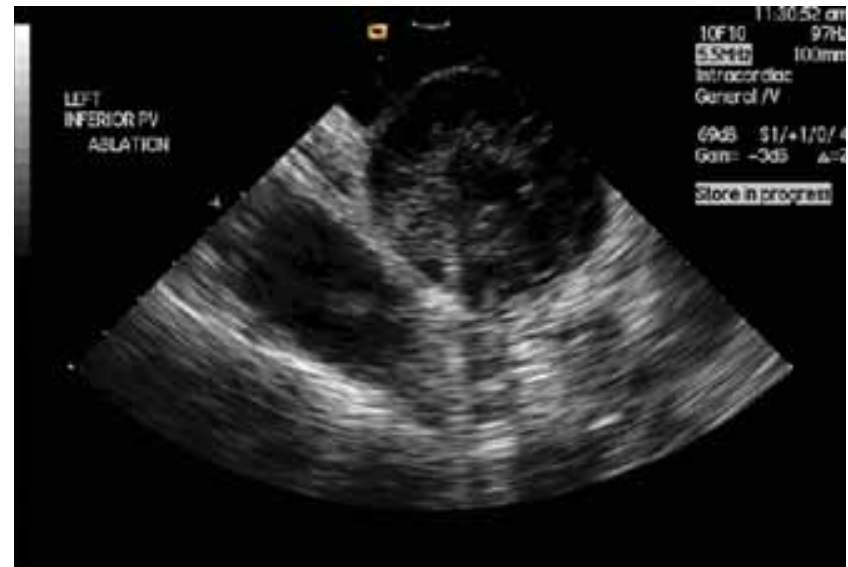

Figure 3: A brisk shower of microbubbles during delivery of radiofrequency energy with an 8 -mm-tip ablation catheter along the inferior border of the LIPV ostium. The microbubbles originate for the catheter tissue interface and spread into the LA cavity. In this situation, radiofrequency energy is immediately terminated. LIPV: left inferior pulmonary vein; LA: left atrial. Reproduced with permission from Europace. ${ }^{125}$

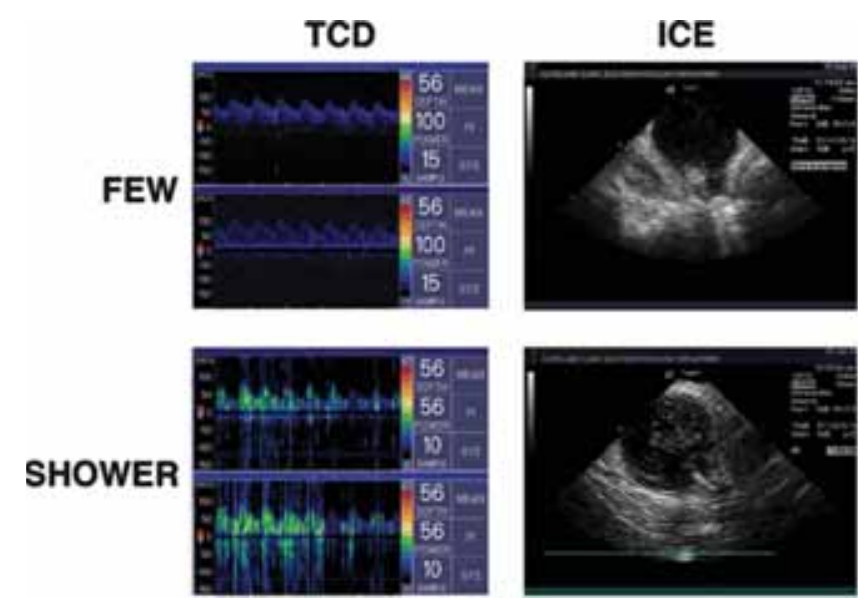

Figure 4: Representative pictures of transcranial Doppler microembolic signals (MESs) and intracardiac echocardiography images. FEW depicts a typical MES (top left) for scattered, non-continuous microbubbles (top right). SHOWER depicts a typical MES (bottom left) for dense, continuous microbubbles (bottom right). Reproduced with permission from the Journal of Cardiovascular Electrophysiology. ${ }^{76}$ 
consequent to detection of SEC in the cardiac chambers on ICE to decrease the risk of left atrial (LA) thrombus formation. ${ }^{84}$ More data on the outcomes of this intervention are needed. Marrouche et al. ${ }^{85}$ showed improved long-term outcomes and reduced risk of pulmonary vein stenosis in patients undergoing cooled tip pulmonary vein isolation when direct visualization of lesion formation and energy delivery monitoring of microbubble formation was done with ICE. ${ }^{85}$ In this study, no stroke or TIA was detected using this strategy, whereas there was a $3 \%$ incidence in patients undergoing ablation without ICE guidance. This speaks to the importance of active ICE use during ablation procedures.

Intraprocedural anticoagulation. Guidelines indicate the need for anticoagulation in the immediate post-sheath insertion period prior to the beginning of transseptal puncture. ${ }^{86,87}$ In a recent analysis, it was shown that higher doses of heparin are needed to achieve therapeutic ACT $(>300)$ in patients with prior anticoagulation with NOACs compared with warfarin. ${ }^{88}$ Typical doses of heparin needed are between 100 and 120 units/ $\mathrm{kg}$ and knowing this difference would help in achieving ACT values prior to the transseptal puncture. Monitoring ACT values should be done every $15 \mathrm{~min}$ at the start of the procedure and carried on every $30 \mathrm{~min}$ after the achievement of therapeutic levels of anticoagulation.

Sheath management. Long sheaths are frequently used for ablation procedures and are the perfect reservoir for blood to stagnate and form linear thrombi. ${ }^{89}$ In addition, removal and introduction of catheters into and out of sheaths offers an opportunity to introduce air and are thus ripe for embolic events. Furthermore, flushing of these sheaths also can cause air to be flushed into the circulation. We feel that excellent laboratory practice should involve the following:

i. Introduction of sheaths over wires carefully for easy maneuverability of the wire during introduction to avoid endothelial damage by scraping vessel walls.

ii. When removing the dilator and wire from the sheath, meticulous attention should be paid to slowly remove these to avoid the creation of vacuum and risk pulling air into the circulation.

iii. Flushing of the sheath should be done with due cognizance of the patients' breathing.

(a) In intubated patients, opening the sheaths to air allows blood to flush out all the air in the system by virtue of positive intrathoracic pressure*

(b) In patients breathing spontaneously, it is important to cover the hub of the sheath, use a syringe to pull out air from the sheath and then use tubing carrying heparinized saline to flush the sheath. It is very important to ensure that the sheath is closed to the atmosphere as the patient may pull in air through the sheath by virtue of spontaneous breaths. ${ }^{* *}$

*However, maintaining an airtight circuit irrespective of mechanical ventilation should be done in an effort to standardize this procedure in laboratory practice.

**The non-return valve at the end of the sheath is mainly to prevent the blood from bleeding back and is not airtight.
The tubing thus needs to be pre-flushed and made free of air bubbles before the procedure. The connection to the sheath side arm also needs to be tapped or agitated in order to dislodge any air bubbles that may be trapped. ${ }^{90}$

iv. Long sheaths should be flushed with heparinized saline for the duration of the procedure at $2 \mathrm{ml} / \mathrm{min}$ or higher rates during the introduction of catheters or their removal.

v. Long sheaths should never be left without catheters in them, especially within the left atrium during AF procedures. This can predispose to thrombus formation, which can easily embolize when a subsequent catheter is introduced.

vi. Prior suctioning the sheaths to ensure the absence of thrombus should also accompany removal of sheaths.

Transseptal puncture. Meticulous attention to detail and frequent checks are required to prevent introduction of embolic material during this procedure. In the presence of visible calcification on the inter-atrial septum on fluoroscopy, trans-septal puncture should be avoided or alternate measures of embolic protection instituted before the trans-septal puncture is attempted. Possible embolic materials include the plastic particles of the inner wall of the transseptal dilator and a core of cardiac tissue into the tip of the septal puncture needle. This is in addition to the omnipresent challenge of excluding air, as mentioned above. ${ }^{91-93}$ Possible measures to prevent scraping of plastic particles include:

i Allowing the needle to rotate within the dilator when inserting the needle;

ii Using needles with larger radii of curvature to avoid interaction with the walls of the dilator;

iii Assembling the needle and dilator outside the body and then introducing the combination into the long sheath;*

iv Repeated insertion and withdrawal of the needle from the dilator outside the body to dislodge possible particles before introduction into the circulation.

*It is important to keep the sheath flushed when assembling the needle-dilator combination outside the body to avoid any thrombus formation in the interim. Once entry into the left atrium is made, an ACT should be checked to ensure adequate anticoagulation and care should be taken henceforth to keep it above therapeutic levels. ${ }^{92}$

Intraprocedural cardioversion. Cardioversion within a procedure has been shown to be associated with increased risks of SCL/SCE formation. This seems intuitive as repeated cardioversion during procedures may provide additional chances for dislodgement of emboli to the brain. In addition, it seems logical to associate the longer length procedures and increased ablation of tissue, for example with scar homogenization and CAFÉ ablation with creation of lines, as additional substrate to increase the risk for SCL/ SCE formation during ablation procedures. ${ }^{94,95}$

Ablation technology. Sources of thromboembolism during ablation include char, steam pops, gaseous bubbles from 
ablation and cavitation phenomena. ${ }^{96,97}$ In addition, the modality of energy used to ablate also determines the chances of thromboembolism. Cryoenergy is thought to be less than irrigated RF energy < non-irrigated RF energy $<$ multielectrode non-irrigated RF energy (PVAC catheters) with respect to the possibility of thromboembolism and rate of SCLs/SCEs during ablation (Figure 5). ${ }^{98,99}$

It is of utmost importance to use a combination of electrogram characteristic modifications such as impedance fall and direct imaging using ICE to monitor lesion formation. It is important to highlight that close temperature monitoring, when using irrigated catheters, is of no use. However, any unexpected rise in the temperature should be viewed with caution as it may suggest some form of blockage in the irrigation ports. In addition, adequate catheter-tissue contact may cause the formation of char at the interface despite irrigation; something the operator must be aware of while ablating. It is also important to realize that ablation itself is a thrombogenic process with the lesions exposing subendothelial collagen which can serve as nidus for thrombus formation. ${ }^{100}$

Furthermore, activation of platelets and the coagulation cascade by the delivery of RF or cryoenergy can induce a prothrombotic state. ${ }^{101}$

Radiofrequency versus cryoablation. The incidence of SCLs/ SCEs has been studied when different types of energy sources were used for AF thermal ablation. Cryoablation and irrigated RFA were found to have similar incidence of SCLs/SCEs in several studies Neumann et al., employed a prospective study design and used T1 and T2 sequences, and along with FLAIR and diffusionweighted MRI to detect SCLs/SCEs before and after atrial AF ablation. This study found no statistically significant difference in incidence of SCLs/SCEs between cryoablation $(8.9 \%)$ versus RFA $(6.8 \%) .{ }^{102}$ Similar results were observed in a study by Siklody et al. ${ }^{14}(4.3 \%$ versus $7.4 \%)$ and Gaita et al. ${ }^{99}(5.6 \%$ and $8.3 \%, \mathrm{p}=0.5)$. Multielectrode phased RF pulmonary vein ablation catheter (PVAC) was associated with increased incidence of SCLs/SCEs when compared to either cryoablation or irrigated RFA. ${ }^{14,103}$

Impact of open versus closed catheter types. Yokoyama et al. ${ }^{104}$ compared ablation lesions between closed loop and open irrigation catheters in canine models and found lower interface temperature, thrombus, and steam pop, especially in low blood flow settings when using open irrigation catheters. This suggested better interface cooling with this technique. ${ }^{104}$ Stabile et al. ${ }^{105}$ also reported a very low complication rate of permanent sequelae in patients undergoing $\mathrm{AF}$ ablation with open irrigation catheters, thus making them a better choice for reducing the impact of neurological consequences during AF ablation.

Takami et al. ${ }^{106}$ noted that during irrigated RF catheter ablation, formation of microbubbles was the greatest during fast saline/contrast injections and steam pops,

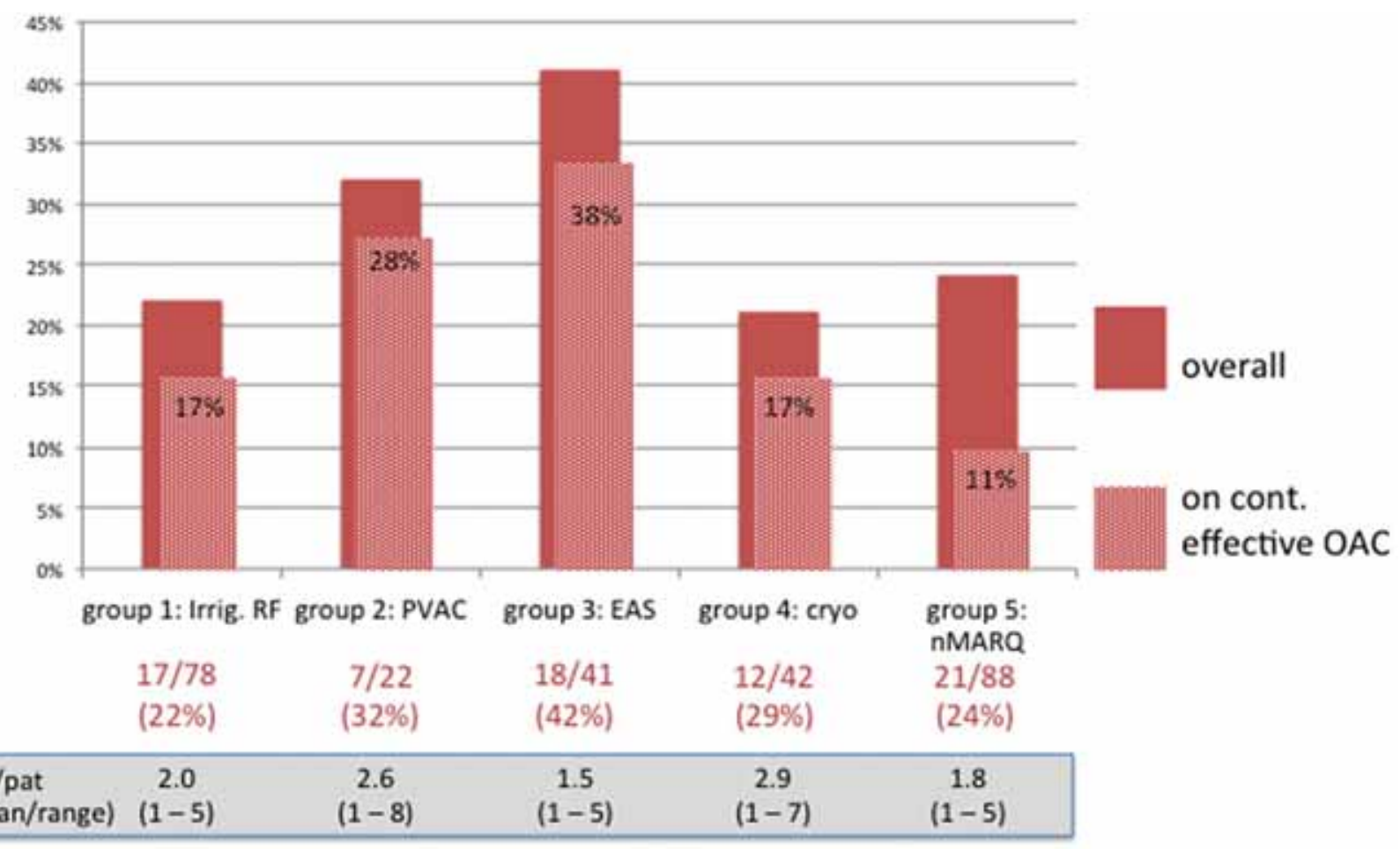

Figure 5: Different SCE rates comparing different ablation technologies using the same MRI sequences and definitions. Relevant reduction for all rates when using periprocedural continued effective oral anticoagulants (warfarin or novel oral anticoagulation agents). SCE: silent cerebral event; MRI: magnetic resonance imaging. Reproduced with permission from the Journal of Cardiovascular Electrophysiology. ${ }^{74}$ 
whereas high-power RF applications drag ablations, and steam pops were more associated with microparticle production. Both microbubbles and microparticles have both been known to increase the risk of MRI-detected SCLs/SCEs. Nguyen et al. ${ }^{107}$ reported an increased incidence of steam pops with high-power open irrigated catheters using 5\% dextrose in water in a perpendicular position compared to other forms of catheter irrigation adding to the data that closed-loop catheters are likely associated with an increased risk of SCLs/SCEs incidence. The use of open irrigated RF catheters also provide a proven advantage of lower complication rates and fewer neurological consequences compared with closed loop design but the operator needs to be wary of the risks of microbubble and microparticle formation with the use of these catheters.

\section{Non-thermal ablation}

Irreversible electroporation. Electroporation involves the use of an external electric field to increase the permeability of the cell plasma membrane. ${ }^{108-110}$ Irreversible electroporation (IRE) has been used as a non-thermal means for tissue destruction. ${ }^{108-111}$ This direct current-based approach has been tested via novel catheters in animal models. ${ }^{112-114}$ This has been used for ablation in pulmonary veins, epicardium, and autonomic ganglia. ${ }^{115,116}$

Being a non-thermal form of ablation, IRE has promise for eliminating the inherent risks of thromboembolic formation. Undoubtedly, there is much innovation that is ongoing and much more remains to be performed prior to implementation in humans. There is a strong need for innovation of electroporation and development of catheter design for adequate endocardial ablation achievement and demonstration of safety as well as the assessment of SCLs/SCEs during ablation.

Novel focused shock wave catheter ablation. Focused shock wave (SW) therapy can create lesions at arbitrary depths without heat generation. Shock wave catheter ablation (SWCA), like electroporation, can theoretically minimize the risk of thrombus formation as a result of tissue coagulation and Joule heat. Hasebe et al. ${ }^{117}$ developed a novel SWCA system that could cause persistent myocardial lesions associated with minimal surface injury and no fatal adverse effects in pigs. In addition, SW therapy being a non-thermal energy may also avoid steam pops that are associated with RF energy and thereby decrease the risk of thrombogenicity and embolism. There is further research required prior to safe employment in clinical use, especially assessment of SCLs/SCEs.

Laser balloon-based pulmonary vein isolation

Dukkipatti et al. ${ }^{118}$ reported similar efficacy with visually guided laser ablation catheter to RFA and no instances of stroke or transient ischemic attack (TIA) in patients undergoing pulmonary vein isolation. ${ }^{118}$ Two separate prospective studies comparing the laser balloon technology to irrigated RF and cryoballoon based pulmonary vein isolation reported no significant difference in the incidence of new SCLs/SCEs between these technologies. ${ }^{18,22}$ However, the number of energy applications was found to be an independent predictor of SCLs/SCEs and the incidence of steam pop and thrombus formation are known to increase with catheter contact force. Innovations focused on newer balloon designs that improves tissue contact and reduce contact force may in fact reduce the risk of new SCLs/SCEs during ablation. $^{22,119}$

\section{Post-procedural measures}

Initiation of anticoagulation. The guidelines have recommend warfarin for a period of 2 months post ablation, irrespective of the individual risk factors after $\mathrm{AF}$ ablation. ${ }^{120,121}$ A variable degree of atrial stunning is noted after the conversion to sinus rhythm. The longer the heart has been in tachycardia, the more the postconversion stunning. This is not attributable to the RF energy delivered, instead is a consequence of the duration of the arrhythmia pre-conversion to sinus rhythm. ${ }^{122}$ In addition, there is also endothelial damage from the ablation that which is thrombogenic.

Studies suggests that post ablation for AF, ischemic strokes most often occur within 2 weeks of the ablation procedure. ${ }^{123}$ At our institution, the following protocol is followed:

$\mathrm{i}$ in patients on uninterrupted warfarin therapy,

(a) if INR is adequate $(>2)$ : we continue therapy with home dose of warfarin;

(b) if INR is inadequate $(<2)$ : we increase the dose of warfarin that night and adjust total dosage to ensure adequate anticoagulation;

ii in patients on therapy with NOAC,

(a) we bridge with unfractionated heparin (UFH) and restart the NOAC the next morning after confirmation of adequate hemostasis;

iii in patients naïve to either warfarin or NOAC,

(a) we start therapy with UFH $4 \mathrm{~h}$ after sheath removal and transition to low molecular weight heparin and warfarin the next morning;

(b) if a NOAC is to start, the UFH is discontinued the next morning after an initial dose of the NOAC.

Restarting dabigatran at $3 \mathrm{~h}$ after the procedure was attempted in a study, where it caused higher bleeding complications than interrupted warfarin therapy. ${ }^{51}$

Continuation of anticoagulation

Guidelines recommend a period of 2 months of anticoagulation. ${ }^{123}$ However, the decision to continue anticoagulation beyond 2 months is left to the discretion of the physician if there is no need for persistent anticoagulation (as in mechanical valves). Studies in AF have shown benefit in continuing anticoagulation indefinitely in patients having higher $\mathrm{CHADS}_{2}$ scores. $^{124}$

\section{Future directions}

It is imperative that a clinically validated protocol for the omission and reinitiation of anticoagulants before, during, and after the ablation procedure be established to minimize the risk of SCLs/SCEs and stroke/TIA. A uniform way of defining and characterizing these lesions and correlating them to meaningful clinical outcomes should be established to be able to accurately assess this risk and quantify the neuro-psychological implications of SCLs/SCEs in 
future prospective randomized clinical trials. The imaging protocols to determine the appropriate timing for evaluating SCLs/SCEs should also be defined as some of these lesions are known to disappear with time. The role of realtime monitoring and intervention based on markers of SCLs/SCEs formation and its influence on subsequent better clinical outcomes may also need to be proven beyond doubt. Further research and innovations to improve existing catheter designs for non-thermal energy sources such as electroporation, SWCA may in fact allow a safer ablation with lower risk of thromboembolism (including SCLs/SCEs).

\section{References}

1. Sheikh A, Patel NJ, Nalluri N, et al. Trends in hospitalization for atrial fibrillation: Epidemiology, Cost, and implications for the future. Prog Cardiovasc Dis. 2015;58(2):105-116.

2. Chugh SS, Havmoeller R, Narayanan K, et al. Worldwide epidemiology of atrial fibrillation: A global burden of disease 2010 study. Circulation. 2014;129(8):837-847.

3. Calkins H, Reynolds MR, Spector P, et al. Treatment of atrial fibrillation with antiarrhythmic drugs or radiofrequency ablation: Two systematic literature reviews and metaanalyses. Circ Arrhythm Electrophysiol. 2009;2(4):349-361.

4. Cheng X, Li X, He Y, et al. Catheter ablation versus antiarrhythmic drug therapy for the management of atrial fibrillation: A meta-analysis. I Interv Card Electrophysiol. 2014;41(3):267-272.

5. Jons C, Hansen PS, Johannessen A, et al. The Medical ANtiarrhythmic Treatment or Radiofrequency Ablation in Paroxysmal Atrial Fibrillation (MANTRA-PAF) trial: Clinical rationale, study design, and implementation. Europace. 2009;11(7):917-923.

6. Kok LC, Mangrum JM, Haines DE, Mounsey JP. Cerebrovascular complication associated with pulmonary vein ablation. J Cardiovasc Electrophysiol. 2002;13(8):764-767.

7. Deneke T, Jais P, Scaglione M, et al. Silent cerebral events/ lesions related to atrial fibrillation ablation: a clinical review. J Cardiovasc Electrophysiol. 2015;26(4):455-463.

8. Herrera Siklódy C, Deneke T, Hocini M, et al. Incidence of asymptomatic intracranial embolic events after pulmonary vein isolation: Comparison of different atrial fibrillation ablation technologies in a multicenter study. J Am Coll Cardiol. 2011;58(7):681-688.

9. Sun X, Lindsay J, Monsein LH, Hill PC, Corso PJ. Silent brain injury after cardiac surgery: A review: Cognitive dysfunction and magnetic resonance imaging diffusion-weighted imaging findings. J Am Coll Cardiol. 2012;60(9):791-797.

10. Haeusler KG, Koch L, Herm J, et al. 3 Tesla MRI-detected brain lesions after pulmonary vein isolation for atrial fibrillation: Results of the MACPAF study. J Cardiovasc Electrophysiol. 2013;24(1):14-21.

11. Deneke T, Shin DI, Balta O, et al. Postablation asymptomatic cerebral lesions: Long-term follow-up using magnetic resonance imaging. Heart Rhythm. 2011;8(11):1705-1711.

12. Haines DE, Stewart MT, Dahlberg S, et al. Microembolism and catheter ablation I: A comparison of irrigated radiofrequency and multielectrode-phased radiofrequency catheter ablation of pulmonary vein ostia. Circ Arrhythm Electrophysiol. 2013;6(1):16-22.

13. Di Biase L, Gaita F, Toso E, et al. Does periprocedural anticoagulation management of atrial fibrillation affect the prevalence of silent thromboembolic lesion detected by diffusion cerebral magnetic resonance imaging in patients undergoing radiofrequency atrial fibrillation ablation with open irrigated catheters? Results from a prospective multicenter study. Heart Rhythm. 2014;11(5):791-798.

14. Gaita F, Leclercq JF, Schumacher B, et al. Incidence of silent cerebral thromboembolic lesions after atrial fibrillation ablation may change according to technology used: Comparison of irrigated radiofrequency, multipolar nonirrigated catheter and cryoballoon. J Cardiovasc Electrophysiol. 2011; 22(9):961-968.

15. Herrera Siklódy C, Deneke T, Hocini M, et al. Incidence of asymptomatic intracranial embolic events after pulmonary vein isolation: Comparison of different atrial fibrillation ablation technologies in a multicenter study. J Am Coll Cardiol. 2011;58(7):681-688.

16. Scaglione M, Caponi D, Anselmino M, et al. Pulmonary vein isolation with a new multipolar irrigated radiofrequency ablation catheter (nMARQ): Feasibility, acute and short-term efficacy, safety, and impact on postablation silent cerebral ischemia. J Cardiovasc Electrophysiol. 2014;25(12): 1299-1305.

17. Verma A, Debruyne P, Nardi S, et al. Evaluation and reduction of asymptomatic cerebral embolism in ablation of atrial fibrillation, but high prevalence of chronic silent infarction: Results of the evaluation of reduction of asymptomatic cerebral embolism trial. Circ Arrhythm Electrophysiol. 2013;6(5):835-842.

18. Wissner E, Metzner A, Neuzil P, et al. Asymptomatic brain lesions following laserballoon-based pulmonary vein isolation. Europace. 2014;16(2):214-219.

19. Lickfett L, Hackenbroch M, Lewalter $T$, et al. Cerebral diffusion-weighted magnetic resonance imaging: a tool to monitor the thrombogenicity of left atrial catheter ablation. J Cardiovasc Electrophysiol. 2006;17(1):1-7.

20. Martinek M, Sigmund E, Lemes C, et al. Asymptomatic cerebral lesions during pulmonary vein isolation under uninterrupted oral anticoagulation. Europace. 2013;15(3): 325-331.

21. Rillig A, Meyerfeldt U, Tilz RR, et al. Incidence and longterm follow-up of silent cerebral lesions after pulmonary vein isolation using a remote robotic navigation system as compared with manual ablation. Circ Arrhythm Electrophysiol. 2012;5(1):15-21.

22. Schmidt B, Gunawardene M, Krieg D, et al. A prospective randomized single-center study on the risk of asymptomatic cerebral lesions comparing irrigated radiofrequency current ablation with the cryoballoon and the laser balloon. J Cardiovasc Electrophysiol. 2013;24(8):869-874.

23. Schrickel JW, Lickfett L, Lewalter T, et al. Incidence and predictors of silent cerebral embolism during pulmonary vein catheter ablation for atrial fibrillation. Europace. 2010; 12(1):52-57.

24. Wieczorek M, Hoeltgen R, Brueck M. Does the number of simultaneously activated electrodes during phased RF multielectrode ablation of atrial fibrillation influence the incidence of silent cerebral microembolism? Heart Rhythm. 2013;10(7):953-959.

25. Wieczorek M, Lukat M, Hoeltgen $R$, et al. Investigation into causes of abnormal cerebral MRI findings following PVAC duty-cycled, phased RF ablation of atrial fibrillation. J Cardiovasc Electrophysiol. 2013;24(2):121-128.

26. Deneke T, Schade A, Müller P, et al. Acute safety and efficacy of a novel multipolar irrigated radiofrequency ablation catheter for pulmonary vein isolation. J Cardiovasc Electrophysiol. 2014;25(4):339-345.

27. Vermeer SE, Longstreth WT, Koudstaal PJ. Silent brain infarcts: A systematic review. Lancet Neurol. 2007;6(7): 611-619. 
28. Gaita F, Corsinovi L, Anselmino M, et al. Prevalence of silent cerebral ischemia in paroxysmal and persistent atrial fibrillation and correlation with cognitive function. J Am Coll Cardiol. 2013; 62(21):1990-1997.

29. Chen LY, Lopez FL, Gottesman RF, et al. Atrial fibrillation and cognitive decline-the role of subclinical cerebral infarcts: The Atherosclerosis Risk in Communities Study. Stroke. 2014;45(9):2568-2574.

30. Medi C, Evered L, Silbert B, et al. Subtle post-procedural cognitive dysfunction after atrial fibrillation ablation. J Am Coll Cardiol. 2013;62(6):531-539.

31. Grosse-Dresselhaus F, Galinovic I, Villringer K, Audebert HJ, Fiebach JB. Difficulty of MRI based identification of lesion age by acute infra-tentorial ischemic stroke. PLoS One. 2014;20;9(3):e92868.

32. Gonzalez RG, Schaefer PW, Buonanno FS, et al. Diffusionweighted MR imaging: Diagnostic accuracy in patients imaged within 6 hours of stroke symptom onset. Radiology. 1999;210(1):155-162.

33. Lovblad $\mathrm{KO}$, Laubach $\mathrm{HJ}$, Baird AE, et al. Clinical experience with diffusion-weighted MR in patients with acute stroke. AJNR Am J Neuroradiol. 1998;19(6):1061-1066.

34. Deneke T, Shin DI, Balta O, et al. Postablation asymptomatic cerebral lesions: long-term follow-up using magnetic resonance imaging. Heart Rhythm. 2011;8(11):1705-1711.

35. Deneke $T$, Nentwich $K$, Schmitt $R$, et al. Exchanging catheters over a single transseptal sheath during left atrial ablation is associated with a higher risk for silent cerebral events. Indian Pacing Electrophysiol J. 2014;14(5):240-249.

36. Stojanov D, Ilic M, Bosnjakovic $\mathrm{P}$, et al. New ischemic brain lesions on diffusion-weighted MRI after carotid artery stenting with filter protection: frequency and relationship with plaque morphology. AJNR Am J Neuroradiol. 2012;33(4):708-714.

37. Gaita F, Caponi D, Pianelli M, et al. Radiofrequency catheter ablation of atrial fibrillation: a cause of silent thromboembolism? Magnetic resonance imaging assessment of cerebral thromboembolism in patients undergoing ablation of atrial fibrillation. Circulation. 2010;122(17):1667-1673.

38. Martinek M, Sigmund E, Lemes C, et al. Asymptomatic cerebral lesions during pulmonary vein isolation under uninterrupted oral anticoagulation. Europace. 2013;15(3):325-331.

39. Verma A, Debruyne P, Nardi S, et al. Evaluation and reduction of asymptomatic cerebral embolism in ablation of atrial fibrillation, but high prevalence of chronic silent infarction: Results of the evaluation of reduction of asymptomatic cerebral embolism trial. Circ Arrhythm Electrophysiol. 2013;6(5):835-842.

40. Medi C, Evered L, Silbert B, et al. Subtle post-procedural cognitive dysfunction after atrial fibrillation ablation. J Am Coll Cardiol. 2013;62(6):531-539.

41. January CT, Wann LS, Alpert JS, et al. 2014 AHA/ACC/ HRS guideline for the management of patients with atrial fibrillation: Executive summary: A report of the American College of Cardiology/American Heart Association Task Force on practice guidelines and the Heart Rhythm Society. Circulation. 2014;130(23):2071-2104.

42. Pepi M, Evangelista A, Nihoyannopoulos P, et al. Recommendations for echocardiography use in the diagnosis and management of cardiac sources of embolism: European Association of Echocardiography (EAE) (a registered branch of the ESC). Eur J Echocardiogr. 2010;11(6):461-476.

43. Sugioka K, Takagi M, Sakamoto S, et al. Predictors of silent brain infarction on magnetic resonance imaging in patients with nonvalvular atrial fibrillation: A transesophageal echocardiographic study. Am Heart J. 2015;169(6):783-790.
44. Martinek M, Sigmund E, Lemes C, et al. Asymptomatic cerebral lesions during pulmonary vein isolation under uninterrupted oral anticoagulation. Europace. 2013;15(3):325-331.

45. Di Biase L, Burkhardt JD, Santangeli P, et al. Periprocedural stroke and bleeding complications in patients undergoing catheter ablation of atrial fibrillation with different anticoagulation management: results from the Role of Coumadin in Preventing Thromboembolism in Atrial Fibrillation (AF) Patients Undergoing Catheter Ablation (COMPARE) randomized trial. Circulation. 2014;129(25):2638-2644.

46. Wazni OM, Beheiry S, Fahmy T, et al. Atrial fibrillation ablation in patients with therapeutic international normalized ratio: Comparison of strategies of anticoagulation management in the periprocedural period. Circulation. 2007;116(22):2531-2534.

47. Hussein AA, Martin DO, Saliba W, et al. Radiofrequency ablation of atrial fibrillation under therapeutic international normalized ratio: A safe and efficacious periprocedural anticoagulation strategy. Heart Rhythm. 2009;6(10) 1425-1429.

48. Di Biase L, Burkhardt JD, Mohanty P, et al. Periprocedural stroke and management of major bleeding complications in patients undergoing catheter ablation of atrial fibrillation: The impact of periprocedural therapeutic international normalized ratio. Circulation. 2010;121(23):2550-2556.

49. Santangeli P, Di Biase L, Horton R, et al. Ablation of atrial fibrillation under therapeutic warfarin reduces periprocedural complications: evidence from a meta-analysis. Circ Arrhythm Electrophysiol. 2012;5(2):302-311.

50. Di Biase L, Gaita F, Toso E, et al. Does periprocedural anticoagulation management of atrial fibrillation affect the prevalence of silent thromboembolic lesion detected by diffusion cerebral magnetic resonance imaging in patients undergoing radiofrequency atrial fibrillation ablation with open irrigated catheters? Results from a prospective multicenter study. Heart Rhythm. 2014;11(5):791-798.

51. Lakkireddy D, Reddy YM, Di Biase L, et al. Feasibility and safety of dabigatran versus warfarin for periprocedural anticoagulation in patients undergoing radiofrequency ablation for atrial fibrillation: Results from a multicenter prospective registry. J Am Coll Cardiol. 2012;59(13): 1168-1174.

52. Lakkireddy D, Reddy YM, Di Biase L, et al. Feasibility and safety of uninterrupted rivaroxaban for periprocedural anticoagulation in patients undergoing radiofrequency ablation for atrial fibrillation: Results from a multicenter prospective registry. J Am Coll Cardiol. 2014;63(10):982-988.

53. Zak M, Castiblanco SA, Garg J, Palaniswamy C, Jacobs LE. Periprocedural management of new oral anticoagulants in atrial fibrillation ablation. J Cardiovasc Pharmacol Ther. 2015; 20(5):457-464.

54. Di Biase L, Lakkireddy D, Trivedi C, et al. Feasibility and safety of uninterrupted periprocedural apixaban administration in patients undergoing radiofrequency catheter ablation for atrial fibrillation: Results from a multicenter study. Heart Rhythm. 2015;12(6):1162-1168.

55. Kim JS, She F, Jongnarangsin K, et al. Dabigatran vs warfarin for radiofrequency catheter ablation of atrial fibrillation. Heart Rhythm. 2013;10(4):483-489.

56. Bassiouny M, Saliba W, Rickard J, et al. Use of dabigatran for periprocedural anticoagulation in patients undergoing catheter ablation for atrial fibrillation. Circ Arrhythm Electrophysiol. 2013;6(3):460-466. 
57. Kaiser DW, Streur MM, Nagarakanti R, Whalen SP, Ellis CR. Continuous warfarin versus periprocedural dabigatran to reduce stroke and systemic embolism in patients undergoing catheter ablation for atrial fibrillation or left atrial flutter. J Interv Card Electrophysiol. 2013;37(3):241-247.

58. Maddox W, Kay GN, Yamada T, et al. Dabigatran versus warfarin therapy for uninterrupted oral anticoagulation during atrial fibrillation ablation. J Cardiovasc Electrophysiol. 2013;24(8):861-865.

59. Providência R, Albenque JP, Combes S, et al. Safety and efficacy of dabigatran versus warfarin in patients undergoing catheter ablation of atrial fibrillation: A systematic review and meta-analysis. Heart. 2014;100(4):324-335.

60. Sardar P, Nairooz R, Chatterjee S, Wetterslev J, Ghosh J, Aronow WS. Meta-analysis of risk of stroke or transient ischemic attack with dabigatran for atrial fibrillation ablation. Am J Cardiol. 2014;113(7):1173-1177.

61. Bin Abdulhak AA, Khan AR, Tleyjeh IM, et al. Safety and efficacy of interrupted dabigatran for peri-procedural anticoagulation in catheter ablation of atrial fibrillation: A systematic review and meta-analysis. Europace. 2013; 15(10):1412-1420.

62. Ichiki $\mathrm{H}$, Oketani $\mathrm{N}$, Ishida $\mathrm{S}$, et al. The incidence of asymptomatic cerebral microthromboembolism after atrial fibrillation ablation: Comparison of warfarin and dabigatran. Pacing Clin Electrophysiol. 2013;36(11):1328-1335.

63. Nakamura K, Naito S, Sasaki T, et al. Silent cerebral ischemic lesions after catheter ablation of atrial fibrillation in patients on 5 types of periprocedural oral anticoagulation-predictors of diffusion-weighted imagingpositive lesions and follow-up magnetic resonance imaging. Circ J. 2016;80(4):870-877.

64. Dillier R, Ammar S, Hessling G, et al. Safety of continuous periprocedural rivaroxaban for patients undergoing left atrial catheter ablation procedures. Circ Arrhythm Electrophysiol. 2014;7(4):576-582.

65. Vamos M, Cappato R, Marchlinski FE, Natale A, Hohnloser SH. Efficacy and safety of rivaroxaban compared with vitamin $\mathrm{K}$ antagonists for peri-procedural anticoagulation in catheter ablation of atrial fibrillation: a systematic review and meta-analysis. Europace.

66. Garg J, Chaudhary R, Krishnamoorthy P, Shah N, Natale A, Bozorgnia B. Safety and efficacy of uninterrupted periprocedural rivaroxaban in patients undergoing atrial fibrillation catheter ablation: A metaanalysis of 1,362 patients. Int J Cardiol. 2016;203:906-908.

67. Kuwahara T. et al. Apixaban versus warfarin for the prevention of periprocedural cerebral thromboembolism in atrial fibrillation ablation: Multicenter prospective randomized study. J Cardiovasc Electrophysiol.

68. Kaps M, Hansen J, Weiher M, Tiffert K, Kayser I, Droste DW. Clinically silent microemboli in patients with artificial prosthetic aortic valves are predominantly gaseous and not solid. Stroke. 1997;28(2):322-325.

69. Clark RE, Brillman J, Davis DA, Lovell MR, Price TR, Magovern GJ. Microemboli during coronary artery bypass grafting. Genesis and effect on outcome. J Thorac Cardiovasc Surg. 1995;109(2):249-257; discussion 257-248.

70. Tinkler K, Cullinane M, Kaposzta Z, Markus HS. Asymptomatic embolisation in non-valvular atrial fibrillation and its relationship to anticoagulation therapy. Eur J Ultrasound. 2002;15(1-2):21-27.

71. Ringelstein EB, Droste DW, Babikian VL, et al. Consensus on microembolus detection by TCD. International Consensus Group on Microembolus Detection. Stroke. 1998;29(3):725-729.

72. Georgiadis D, Lindner A, Manz M, et al. Intracranial microembolic signals in 500 patients with potential cardiac or carotid embolic source and in normal controls. Stroke. 1997;28(6):1203-1207.

73. Stork JL, Kimura K, Levi CR, Chambers BR, Abbott AL, Donnan GA. Source of microembolic signals in patients with high-grade carotid stenosis. Stroke. 2002;33(8):2014-2018.

74. Sauren LD, VAN Belle Y, DE Roy L, et al. Transcranial measurement of cerebral microembolic signals during endocardial pulmonary vein isolation: comparison of three different ablation techniques. I Cardiovasc Electrophysiol. 2009;20(10):1102-1107.

75. Sauren LD, et al. Increased number of cerebral emboli during percutaneous endocardial pulmonary vein isolation versus a thoracoscopic epicardial approach. Eur J Cardiothorac Surg. 2009;36(5):833-837.

76. Kilicaslan F, Verma A, Saad E, et al. Transcranial Doppler detection of microembolic signals during pulmonary vein antrum isolation: Implications for titration of radiofrequency energy. J Cardiovasc Electrophysiol. 2006;17(5):495-501.

77. Nagy-Baló E, Tint D, Clemens M, et al. Transcranial measurement of cerebral microembolic signals during pulmonary vein isolation: A comparison of two ablation techniques. Circ Arrhythm Electrophysiol. 2013;6(3):473-480.

78. Nagy-Balo E, Kiss A, Condie C, Stewart M, Edes I, Csanadi Z. Predictors of cerebral microembolization during phased radiofrequency ablation of atrial fibrillation: Analysis of biophysical parameters from the ablation generator. Heart Rhythm. 2014;11(6):977-983.

79. Nagy-Baló E, Tint D, Clemens M, et al. Transcranial measurement of cerebral microembolic signals during pulmonary vein isolation: A comparison of two ablation techniques. Circ Arrhythm Electrophysiol. 2013;6(3):473-480.

80. Consensus Committee of the Ninth International Cerebral Hemodynamic Symposium. Basic identification criteria of Doppler microembolic signals. Stroke. 1995;26(6):1123.

81. Ringelstein EB, Droste DW, Babikian VL, et al. Consensus on microembolus detection by TCD. International Consensus Group on Microembolus Detection. Stroke. 1998; 29(3):725-729.

82. Dittrich R, Ritter MA, Kaps M, et al. The use of embolic signal detection in multicenter trials to evaluate antiplatelet efficacy: signal analysis and quality control mechanisms in the CARESS (Clopidogrel and Aspirin for Reduction of Emboli in Symptomatic carotid Stenosis) trial. Stroke. 2006;37(4):1065-1069.

83. Wood MA, Shaffer KM, Ellenbogen AL, Ownby ED. Microbubbles during radiofrequency catheter ablation: Composition and formation. Heart Rhythm. 2005;2(4): 397-403.

84. Ren JF, Marchlinski FE, Callans DJ, et al. Increased intensity of anticoagulation may reduce risk of thrombus during atrial fibrillation ablation procedures in patients with spontaneous echo contrast. I Cardiovasc Electrophysiol. 2005;16(5):474-477.

85. Marrouche NF, Martin DO, Wazni O, et al. Phased-array intracardiac echocardiography monitoring during pulmonary vein isolation in patients with atrial fibrillation: Impact on outcome and complications. Circulation. 2003; 107(21):2710-2716.

86. January CT, Wann LS, Alpert JS, et al. 2014 AHA/ACC/ HRS guideline for the management of patients with atrial fibrillation: a report of the American College of Cardiology/ American Heart Association Task Force on practice guidelines and the Heart Rhythm Society. Circulation. 2014; 130(23): e199-267.

87. Calkins H, Kuck KH, Cappato R, et al. 2012 HRS/EHRA/ ECAS expert consensus statement on catheter and surgical ablation of atrial fibrillation: Recommendations for patient 
selection, procedural techniques, patient management and follow-up, definitions, endpoints, and research trial design. Europace. 2012;14(4):528-606.

88. Armbruster HL, Lindsley JP, Moranville MP, et al. Safety of novel oral anticoagulants compared with uninterrupted warfarin for catheter ablation of atrial fibrillation. Ann Pharmacother. 2015;49(3):278-284.

89. Nesbitt WS, Mangin P, Salem HH, Jackson SP. The impact of blood rheology on the molecular and cellular events underlying arterial thrombosis. J Mol Med (Berl). 2006; 84(12):989-995.

90. Blanc JJ, Almendral J, Brignole $\mathrm{M}$, et al. Consensus document on antithrombotic therapy in the setting of electrophysiological procedures. Europace. 2008;10(5):513-527.

91. Csanadi Z, Nagy-Baló E, Danik S, et al. Cerebrovascular complications related to atrial fibrillation ablation and strategies for periprocedural stroke prevention. Cardiac Electrophysiology Clinics. 2014;6(1):111-123.

92. Feld GK, Tiongson J, Oshodi G. Particle formation and risk of embolization during transseptal catheterization: comparison of standard transseptal needles and a new radiofrequency transseptal needle. J Interv Card Electrophysiol. 2011;30(1):31-36.

93. Greenstein E, Passman R, Lin AC, Knight BP. Incidence of tissue coring during transseptal catheterization when using electrocautery and a standard transseptal needle. Circ Arrhythm Electrophysiol. 2012;5(2):341-344.

94. Gaita F, Caponi D, Pianelli M, et al. Radiofrequency catheter ablation of atrial fibrillation: A cause of silent thromboembolism? Magnetic resonance imaging assessment of cerebral thromboembolism in patients undergoing ablation of atrial fibrillation. Circulation. 122(17):1667-1673.

95. Ichiki H, Oketani N, Ishida S, et al. Incidence of asymptomatic cerebral microthromboembolism after atrial fibrillation ablation guided by complex fractionated atrial electrogram. J Cardiovasc Electrophysiol. 2012;23(6):567-573.

96. Kalman JM, Fitzpatrick AP, Olgin JE, et al. Biophysical characteristics of radiofrequency lesion formation in vivo: Dynamics of catheter tip-tissue contact evaluated by intracardiac echocardiography. Am Heart J. 1997;133(1):8-18.

97. Johansen P. Mechanical heart valve cavitation. Expert Rev Med Devices. 2004;1(1):95-104.

98. Sauren LD, VAN Belle Y, DE Roy L, et al. Transcranial measurement of cerebral microembolic signals during endocardial pulmonary vein isolation: Comparison of three different ablation techniques. J Cardiovasc Electrophysiol. 2009;20(10):1102-1107.

99. Gaita F, Leclercq JF, Schumacher B, et al. Incidence of silent cerebral thromboembolic lesions after atrial fibrillation ablation may change according to technology used: Comparison of irrigated radiofrequency, multipolar nonirrigated catheter and cryoballoon. J Cardiovasc Electrophysiol. 2011;22(9):961-968.

100. van Oeveren W, Crijns HJ, Korteling BJ, et al. Blood damage, platelet and clotting activation during application of radiofrequency or cryoablation catheters: A comparative in vitro study. J Med Eng Technol. 1999;23(1):20-25.

101. Pieragnoli P, Gori AM, Ricciardi G, et al. Effects of cryoablation and radiofrequency ablation on endothelial and blood clotting activation. Intern Emerg Med. 2014;9(8):853-860.

102. Neumann T, Kuniss M, Conradi G, et al. MEDAFI-Trial (Micro-embolization during ablation of atrial fibrillation): Comparison of pulmonary vein isolation using cryoballoon technique vs. radiofrequency energy. Europace. 2011;13 (1):37-44
103. Herrera Siklódy C, Deneke T, Hocini M, et al. Incidence of asymptomatic intracranial embolic events after pulmonary vein isolation: comparison of different atrial fibrillation ablation technologies in a multicenter study. J Am Coll Cardiol. 2011;58(7):681-688.

104. Yokoyama K, Nakagawa H, Wittkampf FH, Pitha JV, Lazzara R, Jackman WM. Comparison of electrode cooling between internal and open irrigation in radiofrequency ablation lesion depth and incidence of thrombus and steam pop. Circulation. 2006;113(1):11-19.

105. Stabile G, Bertaglia E, Pappone A, et al. Low incidence of permanent complications during catheter ablation for atrial fibrillation using open-irrigated catheters: A multicentre registry. Europace. 2014;16(18):1154-1159.

106. Takami M, Lehmann HI, Parker KD, Welker KM, Johnson SB, Packer DL. Effect of left atrial ablation process and strategy on microemboli formation during irrigated radiofrequency catheter ablation in an in vivo model. Circ Arrhythm Electrophysiol. 2016;9(1):e003226.

107. Nguyen DT, Olson M, Zheng L, Barham W, Moss JD, Sauer WH. Effect of irrigant characteristics on lesion formation after radiofrequency energy delivery using ablation catheters with actively cooled tips. J Cardiovasc Electrophysiol. 2015;26(7):792-798.

108. Davalos RV, Mir IL, Rubinsky B. Tissue ablation with irreversible electroporation. Ann Biomed Eng. 2005; 33(2):223-231.

109. Lavee J, Onik G, Mikus P, Rubinsky B. A novel nonthermal energy source for surgical epicardial atrial ablation: irreversible electroporation. Heart Surg Forum. 2007;10(2): E162-167.

110. Phillips M, Maor E, Rubinsky B. Nonthermal irreversible electroporation for tissue decellularization. J Biomech Eng. 2010;132(9):091003.

111. Freeman SA, Wang MA, Weaver JC. Theory of electroporation of planar bilayer membranes: predictions of the aqueous area, change in capacitance, and pore-pore separation. Biophys J. 1994;67(1):42-56.

112. Madhavan M, Venkatachalam KL, Swale MJ, et al. Novel percutaneous epicardial autonomic modulation in the canine for atrial fibrillation: Results of an efficacy and safety study. [published ahead of print on February 8, 2016]. Pacing Clin Electrophysiol.

113. DeSimone CV, Kapa S, Asirvatham SJ. Electroporation: Past and future of catheter ablation. Circ Arrhythm Electrophysiol. 2014;7(4):573-575.

114. Wittkampf FH, van Driel VJ, van Wessel $H$, et al. Myocardial lesion depth with circular electroporation ablation. Circ Arrhythm Electrophysiol. 2012;5(3):581-586.

115. van Driel VJ, Neven KG, van Wessel H, et al. Pulmonary vein stenosis after catheter ablation: Electroporation versus radiofrequency. Circ Arrhythm Electrophysiol. 2014;7(4): 734-738.

116. Neven $K$, van Driel $V$, van Wessel $H$, van Es $R$, Doevendans PA, Wittkampf F. Myocardial lesion size after epicardial electroporation catheter ablation after subxiphoid puncture. Circ Arrhythm Electrophysiol. 2014; 7(4):728-733.

117. Hasebe Y, Yamamoto H, Fukuda K, et al. Development of a novel shock wave catheter ablation system-the first feasibility study in pigs. PLoS One. 2015;10(1): e0116017.

118. Dukkipati SR, Neuzil P, Skoda J, et al. Visual balloonguided point-by-point ablation: reliable, reproducible, and persistent pulmonary vein isolation. Circ Arrhythm Electrophysiol. 2010;3(3):266-273. 
119. Yokoyama K, Nakagawa H, Shah DC, et al. Novel contact force sensor incorporated in irrigated radiofrequency ablation catheter predicts lesion size and incidence of steam pop and thrombus. Circ Arrhythm Electrophysiol. 2008;1(5):354-362.

120. European Heart Rhythm Association (EHRA), European Cardiac Arrhythmia Scoiety (ECAS), American College of Cardiology (ACC), et al. HRS/EHRA/ECAS expert Consensus Statement on catheter and surgical ablation of atrial fibrillation: Recommendations for personnel, policy, procedures and follow-up. A report of the Heart Rhythm Society (HRS) Task Force on catheter and surgical ablation of atrial fibrillation. Heart Rhythm. 2007;4(6):816-861.

121. Karasoy D, Gislason GH, Hansen J, et al. Oral anticoagulation therapy after radiofrequency ablation of atrial fibrillation and the risk of thromboembolism and serious bleeding: Long-term follow-up in nationwide cohort of Denmark. Eur Heart J. 2015; 36(5):307-314a.

122. Sparks PB, Jayaprakash S, Vohra JK, et al. Left atrial "stunning" following radiofrequency catheter ablation of chronic atrial flutter. J Am Coll Cardiol. 1998; 32(2):468-475.

123. Oral H, Chugh A, Ozaydin M, et al. Risk of thromboembolic events after percutaneous left atrial radiofrequency ablation of atrial fibrillation. Circulation. 2006;114(8):759-765.

124. Noseworthy PA, Yao X, Deshmukh AJ, et al. Patterns of anticoagulation use and cardioembolic risk after catheter ablation for atrial fibrillation. J Am Heart Assoc. 2015;4(11):pii:e002597.

125. Saliba W, Thomas J. Intracardiac echocardiography during catheter ablation of atrial fibrillation. Europace. 2008;10(Suppl 3):iii42-47. 